\title{
Examination of faecal Bifidobacterium populations in breast- and formula-fed infants during the first 18 months of life
}

Correspondence

Anne L. McCartney

a.l.mccartney@reading.ac.uk

Received 6 July 2010

Revised 9 September 2010

Accepted 12 September 2010

\author{
Laure C. Roger, Adele Costabile, Diane T. Holland, Lesley Hoylest \\ and Anne L. McCartney
}

Microbial Ecology and Health Group, Food Microbial Sciences Unit, Department of Food and Nutritional Sciences, University of Reading, Whiteknights Campus, PO Box 226, Reading RG6 6AP, UK

\begin{abstract}
Bifidobacteria in the infant faecal microbiota have been the focus of much interest, especially during the exclusive milk-feeding period and in relation to the fortification of infant formulae to better mimic breast milk. However, longitudinal studies examining the diversity and dynamics of the Bifidobacterium population of infants are lacking, particularly in relation to the effects of weaning. Using a polyphasic strategy, the Bifidobacterium populations of breast- and formula-fed infants were examined during the first 18 months of life. Bifidobacterium-specific denaturing gradient gel electrophoresis demonstrated that breast-fed infants harboured greater diversity than formula-fed infants and the diversity of the infants' Bifidobacterium populations increased with weaning. Twenty-seven distinctive banding profiles were observed from $\sim 1100$ infant isolates using ribosomal intergenic spacer analysis, 14 biotypes of which were confirmed to be members of the genus Bifidobacterium. Two profiles $(\mathrm{H}$, Bifidobacterium longum subsp. infantis; and I, Bifidobacterium bifidum) were common culturable biotypes, seen in 9/10 infants, while profile $\mathrm{E}$ (Bifidobacterium breve) was common among breast-fed infants. Overall, inter- and intra-individual differences were observed in the Bifidobacterium populations of infants between 1 and 18 months of age, although weaning was associated with increased diversity of the infant Bifidobacterium populations. Breast-fed infants generally harboured a more complex Bifidobacterium microbiota than formula-fed infants.
\end{abstract}

\section{INTRODUCTION}

In the early 1900s, Tissier suggested that the better health status of breast-fed infants was associated with higher numbers of bifidobacteria in their faeces compared with their formula-fed counterparts (Dai \& Walker, 1999). It is now thought that the high levels of bifidobacteria found in the faeces of breast-fed infants may partially explain why they are, in general, less susceptible to gastrointestinal (GI) diseases in the first year of life compared with formula-fed

†Present address: Department of Microbiology, University College Cork, Cork, Ireland.

Abbreviations: DAPI, 4',6-diamidino-2-phenylindole; DGGE, denaturing gradient gel electrophoresis; FISH, fluorescence in situ hybridization; Gl, gastrointestinal; ISR, intergenic spacer region; M, monthly; PW, preweaning; Rf, retardation factor; RISA, ribosomal intergenic spacer analysis; $\mathrm{W}$, weaning.

The GenBank/EMBL/DDBJ accession numbers for the sequences discussed in this paper are listed in Table 2.

Two supplementary figures, showing Bifidobacterium populations present in faecal samples of infants BF4 and BF1 as assessed by $\mathrm{FISH}$ analysis, are available with the online version of this paper. infants (Liepke et al., 2002). In addition, breast-fed infants have significantly lower rates of respiratory and urinary infections and food allergies compared with formula-fed infants (López-Alarcón et al., 1997; Wilson et al., 1998; Harmsen et al., 2000b; Wold \& Adlerberth, 2000; Hoppu et al., 2001; Howie, 2002). Numerous health benefits have been associated with members of the genus Bifidobacterium, including modulation of the immune system (Kirjavainen et al., 2002), vitamin production (Noda et al., 1994), relief of atopic dermatitis symptoms in infants (Isolauri et al., 2000) and reduction of diarrhoea, rotavirus infections and lactose intolerance in children and adults (Saavedra et al., 1994; Jiang et al., 1996).

On average, the Bifidobacterium population of a 2-weekold infant contributes $40-60 \%$ of the total faecal microbiota; however, bifidobacteria can be non-detectable in some formula-fed infants and constitute $90 \%$ of total bacteria in exclusively breast-fed newborns (Harmsen et al., 1999, 2000a). Sakata et al. (2005) identified Bifidobacterium longum subsp. longum as the species most commonly found in 1-month-old infants, irrespective of feeding regime, followed by (in order of predominance) B. longum 
subsp. infantis, B. breve, B. catenulatum, B. adolescentis and $B$. bifidum. Other studies have shown that $B$. breve is the member of the genus Bifidobacterium found most frequently in both breast- and formula-fed infants (Mackie et al., 1999; Matsuki et al., 1999; Fanaro et al., 2003). The discrepancies between studies may be due to different methods having been used, different milk formulae and geographically distinct infant groups. While the predominance of bifidobacteria can vary according to the infant's environment and type of feeding, levels of these bacteria gradually decrease with age (Mitsuoka, 1996). This observation may be explained by the change of diet, withdrawal of breast or formula milk, physiological changes in the growing infant and maturation of the GI tract (Mitsuoka, 1996; Hopkins et al., 2005). It is generally accepted that $B$. adolescentis, $B$. catenulatum and B. longum subsp. longum are characteristic of adult Bifidobacterium populations, whereas B. breve, B. bifidum and B. longum subsp. infantis are more common among infants (Mitsou et al., 2008). However, the transition of the infant Bifidobacterium population (in terms of dynamics and diversity) to the adult Bifidobacterium population in an individual has not yet been described (Matsuki et al., 2004; Dinoto et al., 2006). In addition, the effect of weaning on the diversity of Bifidobacterium populations has not yet been examined fully (McCartney et al., 1996; Klijn et al., 2005; Klaassens et al., 2009).

Investigation into the diversity and dynamics of the predominant Bifidobacterium population of human adults demonstrated that genetic fingerprinting methods allowed good discrimination of different biotypes below species level (McCartney et al., 1996). One subject harboured a simple and relatively stable faecal Bifidobacterium population across the 12-month study, while the second subject harboured a much more complex and dynamic faecal Bifidobacterium population. No common biotypes were identified among the predominant cultivable bifidobacteria of the two donors. The follow-up study from the same research group confirmed the finding that each subject $(n=10)$ harboured a unique predominant Bifidobacterium population (Kimura et al., 1997). More recently, a number of different fingerprinting/profiling methods have been developed, some of which are rather less labour intensive than the ribotyping and pulsed-field gel electrophoresis protocols used by Tannock and colleagues (McCartney et al., 1996; Kimura et al., 1997). One such method is ribosomal intergenic spacer analysis (RISA), which involves restriction fragment length analysis of the ribosomal intergenic spacer region (ISR) of bacterial genomes (Kabadjova et al., 2002). The use of ISR sequences for differentiation between isolates is known to be more useful than $16 \mathrm{~S}$ rRNA gene sequence analysis. For example, $16 \mathrm{~S}$ rRNA gene sequences of species of the genus Bifidobacterium can have $\geqslant 99 \%$ similarity (i.e. B. longum subsp. infantis and B. longum subsp. longum), whereas the ISR gene sequences have greater sequence divergence in closely related species (Ventura et al., 2001, 2006).
Recently, Turroni et al. (2009a) examined the diversity of the Bifidobacterium population in colonic mucosal and faecal samples of humans (infants, adolescents and adults) based on ISR sequences of presumptive isolates of the genus Bifidobacterium. Inter-individual variation was observed, together with differences based on ecological site the isolates were cultured from (mucosal versus faecal samples), although some caution is warranted for the latter, as stool samples were from infants (no details of age and/or diet were provided) and mucosal samples were from adolescent or adult subjects. Interestingly, Turroni et al. (2009a) referred to ' $a$ set of 32 strains which appeared to be commonly distributed among different individuals. The majority of these (21) were only isolated from mucosal samples and often then only from two different subjects (14), although B. breve $246 \mathrm{~B}$ was isolated from mucosa of eight different subjects. Another study from the same group employed microbiomic analysis (i.e. Bifidobacterium-specific 16S rRNA gene libraries) to examine the Bifidobacterium population in colonic mucosal samples from five healthy adults (Turroni et al., 2009b). As well as showing inter-individual variation in the Bifidobacterium populations, novel Bifidobacterium phylotypes were identified, which may reflect the niche investigated and/or the methodology used, although non-specific amplification was also evident. However, it is clear that the diversity of the human gastrointestinal bifidobacterium population has not yet been fully elucidated.

We investigated the development of faecal Bifidobacterium populations in relation to diet and age as part of a longitudinal study of the infant faecal microbiota (Roger \& McCartney, 2010). The aim of this work was to monitor the infant faecal microbiota using a polyphasic approach that allowed examination of Bifidobacterium populations and detection of common or divergent colonization patterns in infants throughout the first 18 months of life.

\section{METHODS}

Bifidobacterium-specific denaturing gradient gel electrophoresis (DGGE). DNA extracted during the longitudinal study by Roger \& McCartney (2010) was used in PCRs with the Bifidobacteriumspecific primers Bif164-f and Bif662-GC-r (Satokari et al., 2001). PCR mixtures comprised $10 \mu \mathrm{l} 5 \times \mathrm{MgCl}_{2}$-free buffer (Promega), $5 \mu \mathrm{l}$ dNTP mix (containing $12.5 \mathrm{mM}$ of each dNTP; Promega), $1 \mu \mathrm{l}$ each of primer Bif164 and primer Bif662-GC-r (both $20 \mathrm{pmol}$ ), $6 \mu \mathrm{MgCl}_{2}$ (25 mM; Promega), $24 \mu \mathrm{l}$ sterile $\mathrm{H}_{2} \mathrm{O}, 1 \mu \mathrm{l}$ GoTaq $\left(1.25 \mathrm{U}^{-1}\right.$; Promega) and $2 \mu$ template DNA. PCR was carried out as described by Satokari et al. (2001). DGGE was carried out as described by Roger \& McCartney (2010), but with minor modifications: the V20-HCDC DGGE system (BDH) was used with gels comprising 45-55\% gradients and run at $85 \mathrm{~V}$.

Ladder for Bifidobacterium-specific DGGE. Type strains of Bifidobacterium species identified as members of the human gut microbiota, namely B. adolescentis DSM $20083^{\mathrm{T}}$, B. bifidum DSM $20456^{\mathrm{T}}$, B. angulatum DSM $20098^{\mathrm{T}}, B$. breve DSM $20213^{\mathrm{T}}, B$. catenulatum DSM $20103^{\mathrm{T}}$, B. gallicum DSM $20093^{\mathrm{T}}$, B. longum subsp. infantis DSM 20088 ${ }^{\mathrm{T}}$, B. longum subsp. longum DSM $20219^{\mathrm{T}}$ and $B$. 
pseudolongum subsp. pseudolongum DSM $20099^{\mathrm{T}}$ were used to develop the Bifidobacterium-specific DGGE ladder. Type strains were grown on pre-reduced Beerens agar (Beerens, 1991) for $48 \mathrm{~h}$ in an anaerobic cabinet (10:10:80, $\mathrm{H}_{2} / \mathrm{CO}_{2} / \mathrm{N}_{2} ;$ MACS 1000 work station, Don Whitley Scientific). A single colony of each was inoculated into pre-reduced supplemented brain heart infusion broth [per litre: $37 \mathrm{~g}$ brain heart infusion broth; $5 \mathrm{~g}$ yeast extract; $4 \mathrm{ml}$ resazurin solution; $0.5 \mathrm{~g}$ L-cysteine; vitamin $\mathrm{K}_{1}\left(1 \mu \mathrm{g} \mathrm{ml}^{-1}\right.$, final concentration); $10 \mathrm{ml}$ haemin solution $\left(50 \mathrm{mg} \mathrm{ml}^{-1}\right)$ ]. After $48 \mathrm{~h}$, the broth cultures were centrifuged at $13792 \mathrm{~g}$ at $4{ }^{\circ} \mathrm{C}$ for $5 \mathrm{~min}$. The supernatants were discarded and DNA was extracted from cell pellets by using the QIAmp DNA Stool Mini kit (Qiagen) according to the manufacturer's instructions. PCR was performed using Bif164-f/Bif662-GC-r as described above. The type strains migrated to five different positions in the Bifidobacterium-specific DGGE. The bands representing B. angulatum DSM $20098^{\mathrm{T}}$ and B. catenulatum DSM $20103^{\mathrm{T}}$ migrated to the same position. Similarly, the bands representing $B$. longum subsp. infantis DSM $20088^{\mathrm{T}}$ and B. longum subsp. longum DSM $20219^{\mathrm{T}}$ migrated to the same position. Bands representing $B$. breve DSM $20213^{\mathrm{T}}$, B. gallicum DSM $20093^{\mathrm{T}}$ and B. pseudolongum subsp. pseudolongum DSM $20099^{\mathrm{T}}$ migrated to approximately the same position. Five type strains were thus selected for the Bifidobacterium-specific DGGE ladder (namely, B. adolescentis DSM $20083^{\mathrm{T}}$, B. bifidum DSM 20456 ${ }^{\mathrm{T}}$, B. catenulatum DSM $20103^{\mathrm{T}}$, B. longum subsp. longum DSM $20219^{\mathrm{T}}$ and B. pseudolongum subsp. pseudolongum DSM $20099^{\mathrm{T}}$ ).

Analysis of DGGE gels. Gel images were imported into TotalLab TL120DM v2006f. Lanes were created manually and background noise was subtracted by using the 'rolling ball' algorithm with a radius of 50 pixels. Automatic band detection was performed with a minimum slope of 100 and a noise reduction of 5. Peaks smaller than $2 \%$ of the highest peak in a lane were discarded. The edge detection method was fixed to a width of 15 for bands in all gels. Detected bands were compared to those on the original, preserved DGGE gels and corrected manually. Horizontal retardation factor (Rf) lines, corresponding to the seven bands in the Bifidobacterium ladder, were used to normalize gels. The same values for all seven $\mathrm{Rf}$ lines were used in all gels to allow comparisons once transferred into the database. Matching analysis was then performed (using a vector of 0.003 ), assessed by eye and corrected manually.

Matching calculations were performed based on the Jaccard similarity coefficient (which matches by the presence/absence of bands), allowing dendrograms to be generated. Pearson correlation coefficient is considered more suitable for highly complex DGGE profiles; however, this algorithm takes into account the whole profile of a lane, including the peak height of each band (van Verseveld \& Röling, 2004). In the case of this study, the Pearson correlation coefficient was not a suitable option as a certain degree of variation (in terms of gradient reproducibility) occurred for every gel developed and the amount of template DNA used in each PCR was not always the same.

Cultivation work. This was done on alternate faecal samples from five breast-fed (BF1-BF5) and five formula-fed (FF1-FF5) infants. For details of the sampling schedule, refer to the paper by Roger \& McCartney (2010), in this issue. Due to the nature of the collection of samples from some infants (where samples were not always available on particular sample weeks), this did not correlate with alternate preweaning (PW), weaning (W) or monthly (M) samples (e.g. PW1, PW3, PW5...).

An aliquot $(500 \mu \mathrm{l})$ of a 1 in $10(\mathrm{w} / \mathrm{v})$ faecal homogenate was added to $4.5 \mathrm{ml}$ pre-reduced peptone water (Oxoid) and used to produce a dilution series $\left(10^{-1}-10^{-8}\right.$ in pre-reduced peptone water). An aliquot $(40 \mu \mathrm{l})$ of each dilution was plated in duplicate onto pre-reduced Beerens agar and incubated in an anaerobic cabinet at $37{ }^{\circ} \mathrm{C}$ for $3-$ 5 days. Colonies were counted from the dilution with 30-300 discrete colonies, and the c.f.u. (g wet weight faeces) ${ }^{-1}$ was determined. Fifteen colonies were randomly selected and streaked to purity on Beerens agar. Pure cultures were stored on Microbank cryogenic beads (ProLab Diagnostics) at $-70{ }^{\circ} \mathrm{C}$.

\section{RISA}

DNA extraction from pure cultures. Ten isolates from each sample for which cultivation work was done were resuscitated on Beerens agar. DNA was extracted from the isolates by using the ChargeSwitch gDNA Mini Bacteria kit following the manufacturer's guidelines.

PCR for RISA. Universal primer 785 (McClellan et al., 1996), which targets a conserved region of the $16 \mathrm{~S}$ rRNA gene, and primer $\mathrm{pR}$ (Leblond-Bourget et al., 1996), which targets a conserved region of the $23 \mathrm{~S}$ rRNA gene, were used to amplify the ISRs ( 1500 bp) of isolates. PCR mixtures comprised $10 \mu \mathrm{l} 5 \times \mathrm{MgCl}_{2}$-free buffer, $5 \mu \mathrm{l}$ dNTP mix, $1 \mu \mathrm{l}$ each of primer 785 and primer pR (both $20 \mathrm{pmol}$ ), $6 \mu \mathrm{MgCl}_{2}, 25 \mu \mathrm{l}$ sterile $\mathrm{H}_{2} \mathrm{O}, 1 \mu \mathrm{l}$ GoTaq and $1 \mu \mathrm{l}$ template. PCR cycles were $94{ }^{\circ} \mathrm{C}$ for $5 \mathrm{~min}$, followed by 30 cycles of $94{ }^{\circ} \mathrm{C}$ for $1 \mathrm{~min}$, $53{ }^{\circ} \mathrm{C}$ for $1 \mathrm{~min}, 72{ }^{\circ} \mathrm{C}$ for $2 \mathrm{~min}$, with a final elongation at $72{ }^{\circ} \mathrm{C}$ for $10 \mathrm{~min}$. Products were examined by using agarose gel electrophoresis and stored at $-20{ }^{\circ} \mathrm{C}$ until required.

Enzymic digestion of amplicons. To select the best array of restriction enzymes for DNA digestion, sequences from GenBank for the type strains of different species of the genus Bifidobacterium were digested in silico using different restriction enzymes (http:// rebase.neb.com/rebase/rebase.html). The following type strains were used: B. breve ATCC $15700^{\mathrm{T}}$ (GenBank accession nos M58731 and M84776), B. adolescentis ATCC $15703^{\mathrm{T}}$ (M58729), B. longum subsp. longum ATCC $15707^{\mathrm{T}}$ (M58739 and M84781), B. bifidum ATCC $29521^{\mathrm{T}}$ (M38018 and M84777), B. longum subsp. infantis ATCC $15697^{\mathrm{T}}$ (X70947 and M84783), B. catenulatum ATCC $27539^{\mathrm{T}}$ (M58732 and M84784), B. angulatum ATCC $27535^{\mathrm{T}}$ (M84775) and B. pseudocatenulatum ATCC $27919^{\mathrm{T}}$ (M84785). Seven enzymes (AluI, TaqI, HpaII, NdeII, BamHI, Sau96I and BstNI) were identified as giving good differentiation of Bifidobacterium species on the basis of the in silico analysis. B. adolescentis DSM $20083^{\mathrm{T}}$, B. angulatum DSM $20098^{\mathrm{T}}$, B. breve DSM $20213^{\mathrm{T}}$, B. catenulatum DSM $20103^{\mathrm{T}}, B$. gallicum DSM 20093 ${ }^{\mathrm{T}}$, B. longum subsp. infantis DSM 20088 ${ }^{\mathrm{T}}, B$. longum subsp. longum DSM $20219^{\mathrm{T}}$ and B. pseudolongum subsp. pseudolongum DSM $20099^{\mathrm{T}}$ were grown on pre-reduced Beerens agar and DNA was extracted from the strains using the ChargeSwitch gDNA Mini Bacteria kit, as described above. PCR was done using primers $\mathrm{pR} / 785$, and products were digested with each of the seven restriction enzymes. Each restriction mixture contained $10 \mathrm{U}$ restriction enzyme, $2 \mu \mathrm{l}$ buffer, $0.2 \mu \mathrm{BSA}, 10 \mu \mathrm{l}$ PCR product and sterile distilled $\mathrm{H}_{2} \mathrm{O}$ to give a final volume of $20 \mu$ l. Digestions were performed for $1.5 \mathrm{~h}$ at 37 or $65{ }^{\circ} \mathrm{C}$, according to the manufacturer's recommendations. Restriction products were separated by electrophoresis using $2.5 \%$ agarose gels in $1 \times$ TAE buffer for $3 \mathrm{~h}$, which were stained by using ethidium bromide $\left(0.5 \mathrm{ng} \mathrm{ml}^{-1}\right)$ for $30 \mathrm{~min}$. After destaining ( $5 \mathrm{~min}$ ), the bands were visualized under UV light (Genesnap, Syngene). HpaII was selected for the digestion of samples as it gave the greatest differentiation (data not shown).

Analysis of RISA gels. Each profile observed was associated with a single letter and a summary table (Table 1) was organized to illustrate all profiles observed per infant and per sample. Analysis was based both on analysis with TotalLab TL120 v2006f software and visual inspection. The table was then used to detect any common profiles and to observe isolate trends and similarities among infants.

Sequencing and identification of representative RISA biotypes. PCR amplification of the $16 \mathrm{~S}$ rRNA gene of selected isolates was done using the universal primers $\mathrm{pH}^{*}\left(5^{\prime}\right.$-AAGGAGGTGATCCAGCCGCA-3') 
and arit (5'-CTGGCTCAGGATGAACGCTG-3'). Almost-complete $(>1350 \mathrm{nt})$ 16S rRNA gene sequences were obtained via the University of Reading's Biocentre. The 16S rRNA gene sequences were subjected to WU-Blast2 searches via the EMBL website (http:// www.ebi.ac.uk/) to establish the identity of the strains. Isolate identities were confirmed by comparison with type-strain sequences within an in-house database. Sequences of the faecal isolates were proofread manually against type-strain sequences, and submitted to GenBank/ EMBL/DDBJ for assignment of accession numbers. All sequences were edited via 4Peaks version 1.7.2 (by A. Griekspoor and Tom Groothuis, http://www.mekentosj.com).

\section{RESULTS AND DISCUSSION}

\section{Bifidobacterium-specific DGGE}

In order to monitor the composition of Bifidobacterium populations of infants over time, PCR-DGGE was done with the Bifidobacterium-specific primers described by Satokari et al. (2001). However, Bifidobacterium amplicons were not always obtained with these primers (and generally correlated with lower Bif164 counts), although universal DGGE profiles were obtained for all DNA samples (Roger \& McCartney, 2010). PCR amplification problems were most frequently observed with $\mathrm{PW}$ samples from formulafed infants. In general, this was consistent with lower Bif164 counts (Roger \& McCartney, 2010) and therefore most likely reflects lower copy numbers of Bifidobacterium $16 \mathrm{~S}$ rRNA genes in these samples. However, a more rigorous DNA extraction method may have enabled detection of the Bifidobacterium population even in those samples with lower levels. Salonen et al. (2010) compared four different faecal DNA extraction methods and demonstrated marked differences in the yields obtained, as well as the relative proportions of certain Gram-positive phyla in community profiling analysis.
A marker was created to help with the analysis of DGGE profiles and to allow presumptive identification of bands in the infant profiles (Fig. 1). DGGE analysis of the type strain profiles demonstrated a single band for all Bifidobacterium species, except for $B$. adolescentis, which displayed three bands (Fig. 1). This was consistent with the findings of Satokari et al. (2001). Bifidobacterium-specific DGGE did not differentiate all the type strains. B. longum subsp. longum and $B$. longum subsp. infantis displayed the same DGGE profiles, as did $B$. angulatum and B. catenulatum. B. breve and B. gallicum produced DGGE profiles with a very faint, fuzzy band (Fig. 1, lanes 29 and 30); this phenomenon was also observed by Satokari et al. (2001).

TotalLab TL120DM v2006f software was employed to analyse the DGGE profiles for each sample obtained from the breast- and formula-fed infants. The resulting dendrogram(s) did not demonstrate any specific clustering in relation to diet or age (data not shown). Consequently, the data were reanalysed in relation to presence/absence of each observed band. In order to achieve this, a composite profile was generated and the DGGE data were transformed into figures illustrating the presence/absence of bands (Figs 2 and 3 ).

DGGE for breast-fed infants. Two samples from the breast-fed group did not produce an amplicon using the Bifidobacterium-specific DGGE primers. These were PW6 from BF4, which corresponded to the course of antibiotics received by the infant and a dramatic drop in Bif164 counts (Supplementary Fig. S1, available with the online version of this paper) and sample M2 from BF6.

During the PW phase, BF2 and BF6 each demonstrated a single DGGE profile comprising two bands (Fig. 2a). On the whole, the PW phase bifidobacterium-specific DGGE profiles of breast-fed infants displayed between one and

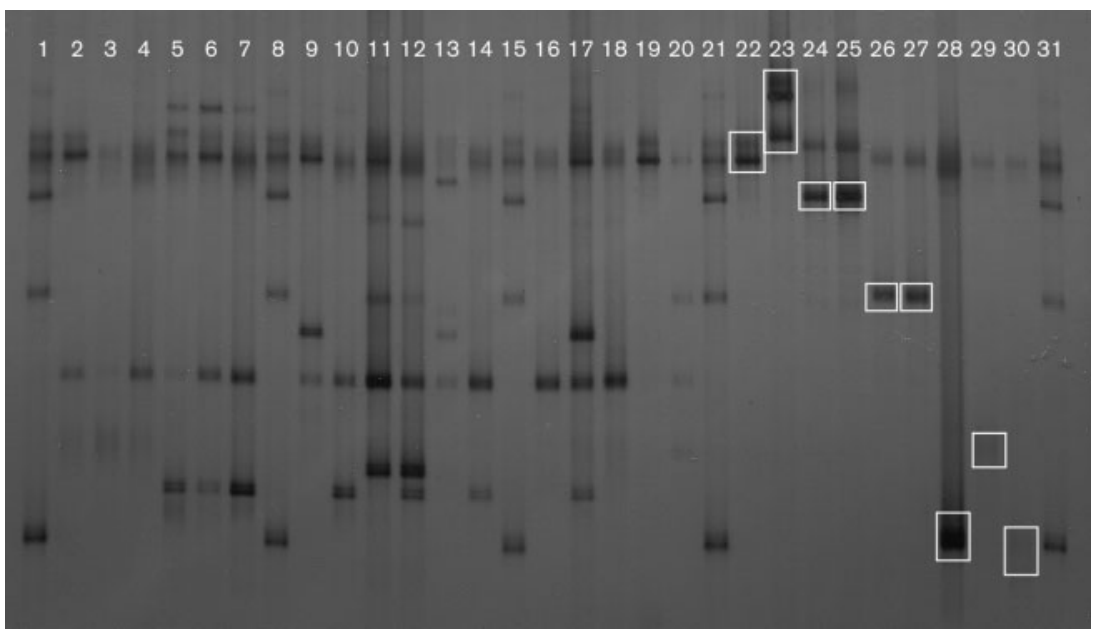

Fig. 1. Composite Bifidobacterium-specific DGGE representing the different profiles observed in samples from breast- and formula-fed infants. Lanes: 1, five-strain marker; 2, BF1 month 10; 3, BF2 month 8; 4, BF2 month $18 ; 5$, BF3 month $12 ; 6$, BF3 month 15 ; 7, BF4 month $15 ; 8$, five-strain marker; 9 , BF5 month $15 ; 10$, BF6 month $15 ; 11$, BF7 month 9; 12 , BF7 month 10; 13, FF1 month 12; 14 , FF2 month 10; 15, five-strain marker; 16, FF3 month $10 ; 17$, FF6 month $10 ; 18$, FF7 month $15 ; 19$, FF4 week 36; 20, BF5 week 26; 21, five-strain marker; 22, B. bifidum; 23, $B$. adolescentis; 24, B. angulatum; 25, $B$. catenulatum; 26, B. longum subsp. longum; $27, B$. longum subsp. infantis; $28, B$. pseudolongum subsp. pseudolongum; 29, B. breve; 30, B. gallicum; 31, five-strain marker. White boxes indicate bands taken as being representative of the respective type strains. 

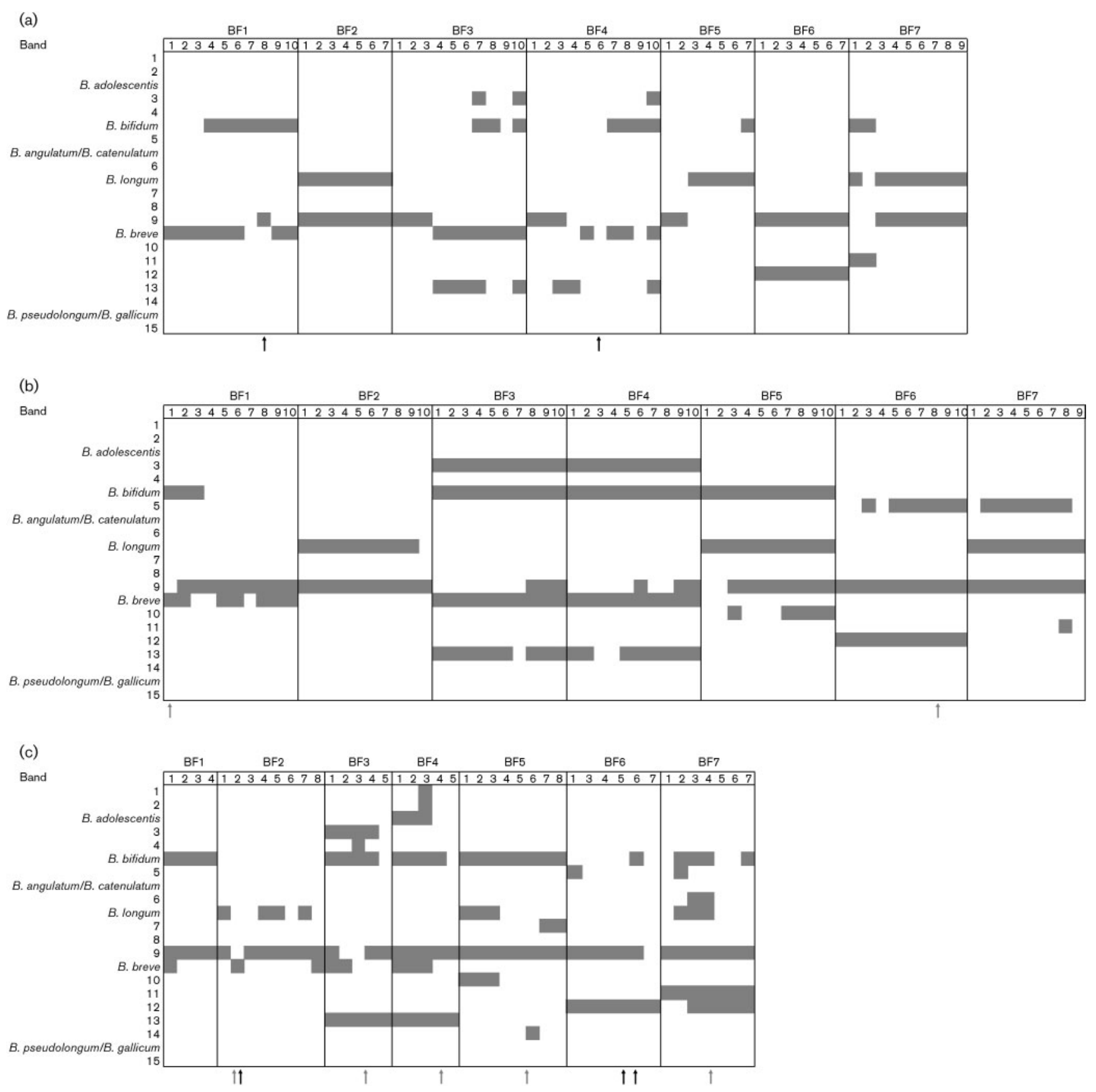

Fig. 2. Representation of Bifidobacterium-specific DGGE profiles for breast-fed infants during the PW (a), W (b) and M (c) phases of feeding. The presence of a band at a particular sampling time is indicated by grey shading. Samples are presented in chronological order (left to right). Black arrows, administration of antibiotics; grey arrows, withdrawal of breast milk.

four bands (with a mean number of bands per profile of 1.8). Five of the seven breast-fed infants displayed a band consistent with that of the B. bifidum band in the marker at some point during the PW phase (BF2 and BF6 did not). Three breast-fed infants showed bands consistent with the B. longum band in the majority of their PW samples (BF2, BF5 and BF7). The profiles from BF1, BF3 and BF4 showed a band consistent with the $B$. breve band in the marker during the PW phase. No bands corresponding to the $B$. adolescentis and $B$. pseudolongum subsp. pseudolongum/B. gallicum bands were seen in the PW profiles of breast-fed infants. It appeared that band 9 was a common band amongst the breast-fed infants (i.e. seen in all infants at some time during each dietary phase). 

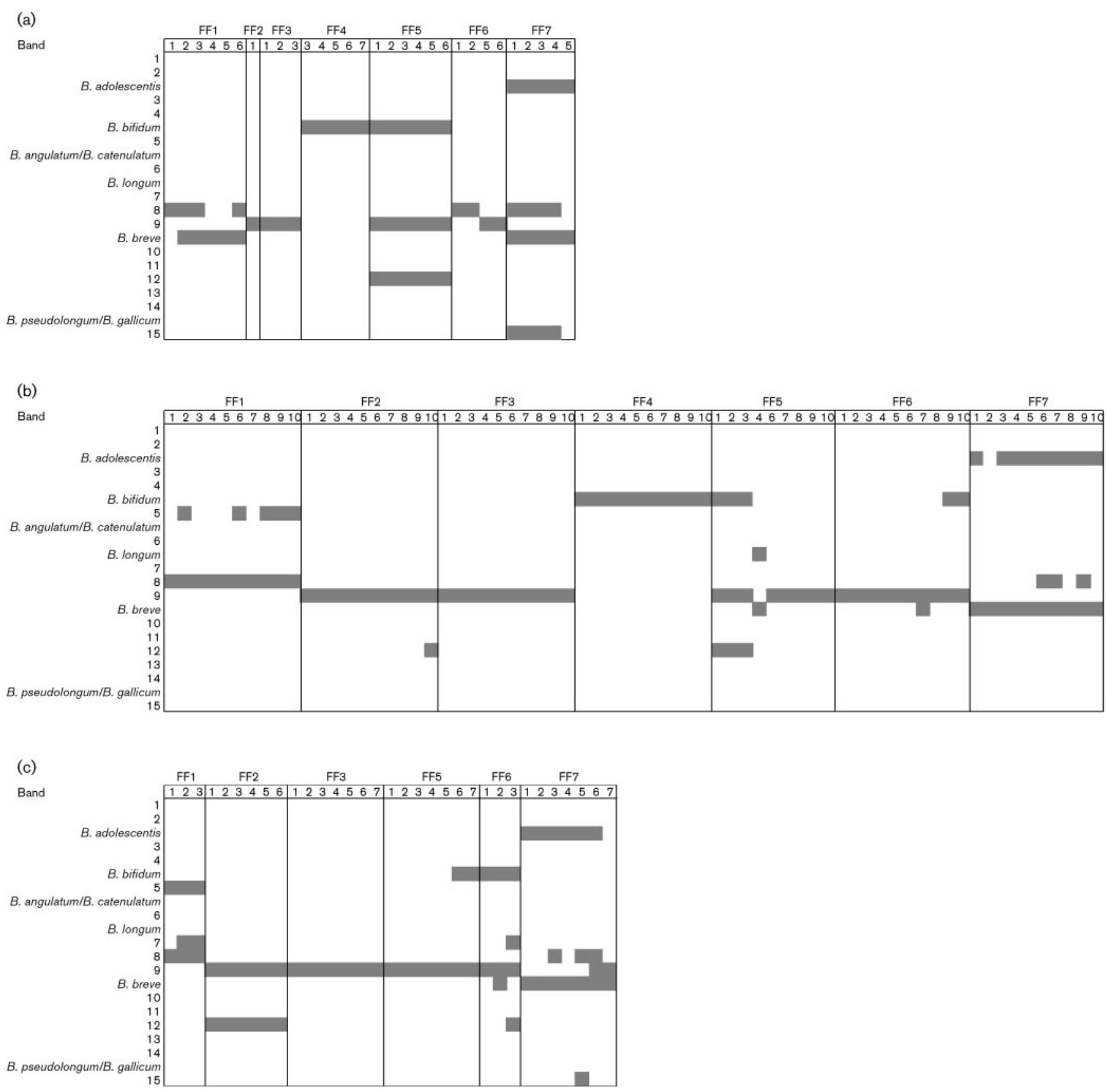

Fig. 3. Representation of Bifidobacterium-specific DGGE profiles for formula-fed infants during the PW (a), W (b) and M (c) phases of feeding. The presence of a band at a particular sampling time is indicated by grey shading. Samples are presented in chronological order (left to right).

The profiles obtained during the $\mathrm{W}$ phase showed similar diversity to those observed during the PW phase (Fig. $2 \mathrm{~b})$, although the profiles of BF5, BF6 and BF7 had an additional band compared with the PW phase and BF7 no longer had a band consistent with B. bifidum. Furthermore, the profiles of the twins (BF3 and BF4) and BF5, BF6 and BF7 generally contained more bands in the $\mathrm{W}$ phase than seen in the PW phase. This was reflected in the fact that the mean number of bands per profile was 3.0 during the $\mathrm{W}$ phase. Overall the profiles of each infant appeared more stable during the $\mathrm{W}$ phase. A band consistent with $B$. bifidum was present in the profiles of four infants (BF7 being the infant who displayed this band during PW but not $W$ ). Bands consistent with $B$. longum and $B$. breve were present in the profiles of the same infants who displayed these bands 
during PW. Band 9 was again a common band to the profiles of breast-fed infants.

The profiles obtained during the $\mathrm{M}$ phase contained between three and seven bands (Fig. 2c), and showed greater diversity than seen for the PW and W phases (with a novel band seen for six of the seven infants; i.e. a band not previously displayed in the profiles). However, the mean number of bands per profile was consistent with the $\mathrm{W}$ phase (i.e. 3.0). Band 9 was again common to the Bifidobacterium-specific DGGE profiles of breast-fed infants (only absent from the profiles of four of the $43 \mathrm{M}$ phase samples). BF2 was the only infant that did not display a band corresponding with the B. bifidum band, but a band consistent with $B$. breve was seen, although only one sample (M6) from BF6 displayed a band consistent with $B$. bifidum. Bands consistent with $B$. longum and $B$. breve were present in the profiles of the same infants who displayed these bands during PW. Bands consistent with $B$. adolescentis were evident in the first three $\mathrm{M}$ phase samples of BF4.

There was no obvious effect of withdrawal of breast-milk or administration of antibiotics on the Bifidobacteriumspecific DGGE profiles of individuals. BF3 and BF4 (twins) displayed a simpler bifidobacterium-specific DGGE profile after breast-feeding ceased, but only one further sample was available, so no conclusions can be drawn from this observation.

DGGE for formula-fed infants. As mentioned above, Bifidobacterium-specific DGGE primers did not amplify a product from some samples. Overall, the PW phase Bifidobacterium-specific DGGE profiles of formula-fed infants appeared to be relatively simple, with between one and four bands present (Fig. 3a). The mean number of bands per profile was 1.9. Bands consistent with $B$. bifidum and $B$. breve were present in two infants (FF4 and FF5, and FF1 and FF7, respectively). Bands consistent with $B$. adolescentis were seen in all PW phase profiles of FF7. Band 9 was present in the PW phase profiles of four infants (FF2, FF3, FF5 and FF6). Overall, the Bifidobacteriumspecific DGGE profiles of formula-fed infants were relatively stable during the $\mathrm{PW}$ phase.

The Bifidobacterium-specific DGGE profiles of formula-fed infants remained relatively simple during the $\mathrm{W}$ phase, although alterations in banding profiles were seen for some individuals (Fig. 3b). Between one and five bands were detected and the profiles from three infants (FF2, FF3 and FF4) displayed a single band throughout the $\mathrm{W}$ phase. A mean number of 1.4 bands were seen per profile for the $\mathrm{W}$ phase samples of formula-fed infants. This was notably less than that seen for breast-fed infants at the same stage. Similar to PW phase profiles, bands consistent with $B$. adolescentis were only seen for FF7, together with a band consistent with $B$. breve and band 8 (on some occasions). A band consistent with $B$. breve was also present in FF5 and FF6, neither of whom displayed this band in their PW profiles. FF5 also contained a band consistent with $B$. longum in sample W4. A band corresponding with $B$. bifidum was seen in three infants (FF4-FF6); FF4 and FF5 also displayed this band in their PW profiles. Band 9 formed the backbone of the Bifidobacterium-specific DGGE profiles of FF2, FF3, FF5 and FF6.

The $M$ phase Bifidobacterium-specific DGGE profiles of formula-fed infants remained simple (Fig. 3c), with between one and five bands per profile (mean 1.9 bands per profile). No Bifidobacterium-specific PCR products were obtained for FF4 from $M$ phase samples. FF3 maintained a Bifidobacterium-specific DGGE profile comprising only band 9 (consistent with the $\mathrm{PW}$ and $\mathrm{W}$ phase profiles). Indeed, FF2 and FF3 maintained the same profile as seen at the end of the $\mathrm{W}$ phase. Band 9 was common among the formula-fed infants, with only FF1 not showing it in M phase DGGE profiles. Bands consistent with $B$. adolescentis were still present in FF7's profiles. The band consistent with $B$. bifidum remained in the profiles of FF5 and FF6, while a band corresponding with $B$. breve was present in FF6 and FF7.

Comparison of DGGE profiles between feeding groups. Overall, the Bifidobacterium profiles were relatively simple (comprising between 1 and 6 bands), in accordance with data described by Klaassens et al. (2009), which showed that the Bifidobacterium populations of two breast-fed and three formula-fed infants were relatively simple and stable between 6 and 11 months of age. However, Klaassens and colleagues observed less stability in the Bifidobacterium profiles of formula-fed infants, most likely reflecting the dietary intervention (prebiotic-supplemented formula). The main difference observed in the current study between the profiles from the breast- and formula-fed infants was the mean number of bands per profile for each dietary phase. Overall, the mean number of bands per breast-fed infant profile was greater than that for the formula-fed profiles (in all dietary phases). In both feeding groups, the mean number of bands per profile increased across the three dietary phases. The Bifidobacteriumspecific DGGE profiles of breast-fed infants varied more during the study than those of formula-fed infants (which were relatively stable over time).

Bands consistent with $B$. adolescentis were observed in the profiles of FF7 across all dietary phases and in some $M$ phase profiles of BF4. Bands corresponding to B. bifidum and $B$. breve were observed in profiles of both feeding groups across the study, while bands corresponding to $B$. longum were observed in profiles of breast-fed infants (throughout the study), but only in the profile of FF5's sample W4. Interestingly, band 9 was seen in both feeding groups and was present throughout the study.

Overall, the diversity of the Bifidobacterium populations harboured by breast-fed infants was greater than that of formula-fed infants from 4 weeks of age and increased with weaning. The formula-fed group showed relatively simple and stable Bifidobacterium-specific DGGE profiles. 


\section{Comparison of DGGE and FISH data}

BF1 experienced a drop in Bif164 counts at sample W3 (week 29, Supplementary Fig. S2, available with the online version of this paper) that corresponded with the disappearance of $B$. bifidum from the Bifidobacteriumspecific DGGE profiles (Fig. 2b). Bif164 counts were relatively stable for the twins (BF3 and BF4) until the start of the M phase, with the exception of BF4's PW6 sample (this correlated with antibiotic administration); interestingly, no amplicon was obtained for Bifidobacteriumspecific PCR-DGGE for this sample. Bifidobacteriumspecific DGGE showed intra-individual variability in the twins' PW and $\mathrm{M}$ phase profiles. In addition, Bifidobacterium-specific PCR was generally unsuccessful for PW samples from FF2, which corresponded with the lowest Bif164 counts of all infants seen during PW. Interestingly, this also correlated with a lack of isolation of any Bifidobacterium strains (discussed below) from the FF2 PW samples for which cultivation work was performed.

Taken together, these results indicated that data generated from the FISH and Bifidobacterium-specific DGGE assays generally complemented each other. Furthermore, they confirmed that the dynamics of the bacterial population was not always discernible via FISH counts. Interestingly, BF7 showed the greatest diversity in the Bifidobacteriumspecific DGGE profiles, despite generally having the lowest Bif164 counts of the infants included in the breast-fed group.

\section{Investigation into the predominant culturable biotypes}

Fifteen isolates were randomly selected from Beerens plates, when available, from the dilution that resulted in discrete colonies. The objective was to genetically fingerprint 10 randomly selected isolates; 15 were subcultured to allow for loss of strains, due to either unsuccessful subculturing (no growth) or inability to resuscitate after cryogenic storage. In total, isolation work was performed for 123 samples from the 10 infants. On nine occasions, no bacterial colonies were obtained from the dilution series [twice from breast-fed infants' $M$ phase samples (BF1 and BF5), three times from PW samples of FF2, once from a W phase sample of FF2 and three times from formula-fed infants' $M$ phase samples (FF3 and FF4)]. In total, genetic fingerprinting was completed for approximately 1100 isolates.

Initial work comprised screening different restriction enzymes using the type strains of nine Bifidobacterium species to determine the relative discriminative power of each enzyme using the amplified 16S-23S rRNA ISR (data not shown). HpaII digests demonstrated the greatest differentiation; however, B. angulatum and B. catenulatum, and $B$. longum subsp. longum and $B$. longum subsp. infantis could not be differentiated (Fig. 4a).

Twenty-seven distinctive banding profiles were identified from the infant isolates (Fig. 4b). In general, the infants appeared to harbour relatively simple predominant Bifidobacterium populations (with between one and three distinct
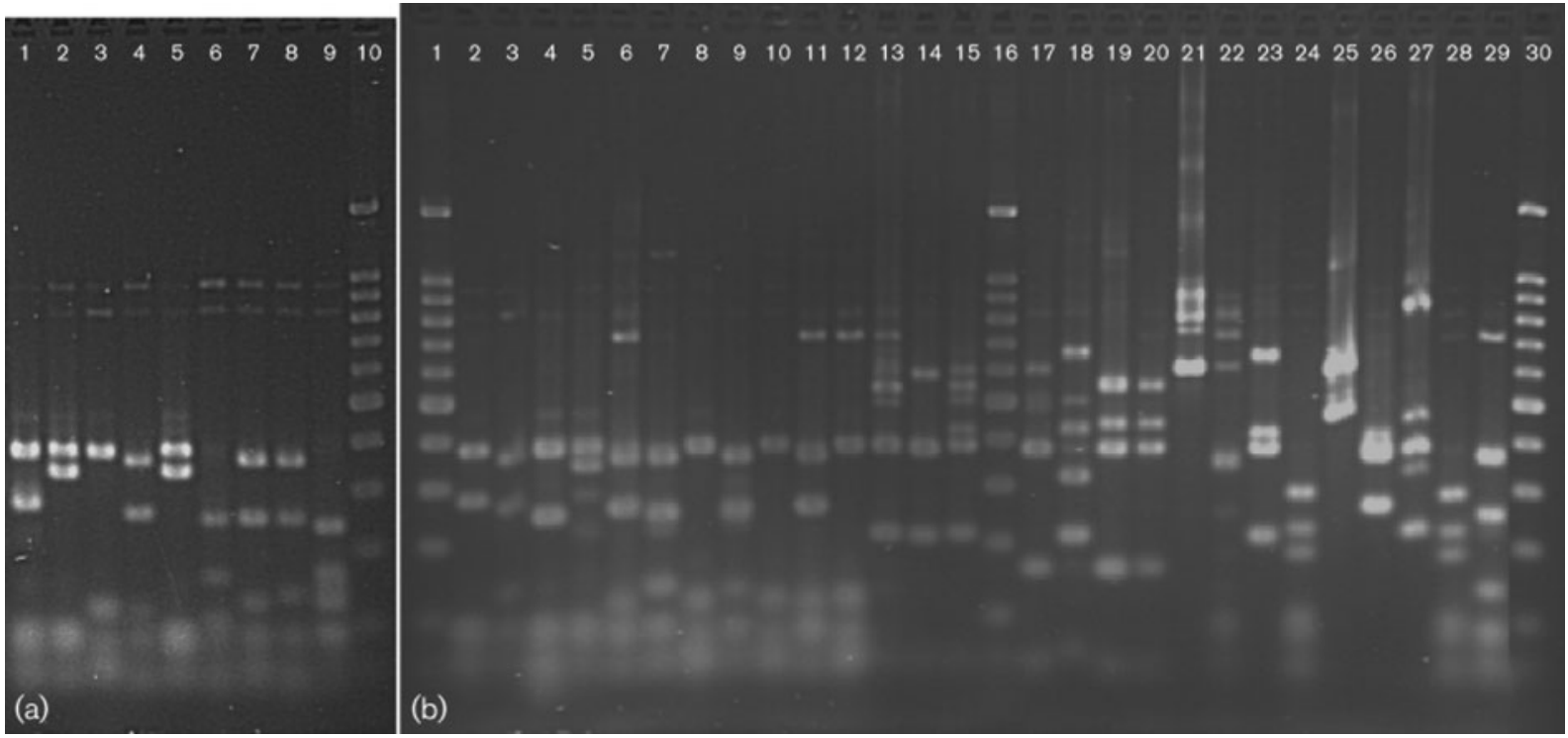

Fig. 4. Genetic fingerprinting of bacterial pure cultures using RISA. (a) Banding profiles of type strains of members of the genus Bifidobacterium obtained by Hpall digestion of amplified 16S-23S ISR. Lanes: 1, B. adolescentis; 2, B. angulatum; 3, B. bifidum; 4, B. breve; 5, B. catenulatum; 6, B. gallicum; 7, B. longum subsp. infantis; 8, B. longum subsp. longum; 9 , B. pseudolongum subsp. pseudolongum; 10, 100 bp ladder. (b) Representative banding profiles obtained by Hpall digestion of amplified 16S-23S ISR of bacteria isolated from infants. Lanes: 1, 100 bp ladder; 2-15, A-N, respectively; 16,100 bp ladder; 17-28, O-Z, respectively; 29, AA; 30, 100 bp ladder. 
biotypes seen per sample) (Table 1). BF3 and BF4 (the twins) displayed the most complex and dynamic Bifidobacterium microbiota of all infants, with more than three biotypes seen on a number of occasions. A mean of 6.7 distinctive biotypes were found per infant during the study: 7.2 per breast-fed infant and 6.2 per formula-fed infant.

A representative isolate was selected for each of the 27 profiles and almost-complete 16S rRNA gene sequences were obtained to allow identification of isolates (Table 2). Fourteen of the 27 biotypes were confirmed to be members of the genus Bifidobacterium, namely profiles A-K, V, Z and AA. The other profiles included Carnobacterium divergens (profile O), Clostridium baratii (W), Clostridium clostridiforme (X), Clostridium innocuum $(\mathrm{Y})$, Collinsella aerofaciens (T), Dorea longicatena (S), Enterococcus faecalis (Q and R), Lactobacillus casei (M), Lactobacillus delbrueckii (P),
Lactobacillus plantarum (U) and Staphylococcus pasteurii (L and $\mathrm{N}$ ). Of these 13 biotypes, only profiles $\mathrm{L}$ and $\mathrm{M}$ were isolated from more than one sample (Table 1).

Profiles H (B. longum subsp. infantis) and I (B. bifidum) were common biotypes, seen in nine of 10 infants (only FF3 did not display profile $\mathrm{H}$ and FF2 did not display profile I). Profile E (B. breve) was common among the breast-fed infants, although FF1 also displayed this biotype. Profiles A, B, C, E, F, H, I and M (of which profile $M$ was not a Bifidobacterium species) were observed during all three dietary phases (PW, W and M). Sixteen profiles (of which profiles D, G, V, Z and AA were Bifidobacterium species) were only observed on one occasion, i.e. from one sample of one infant, although profiles D (B. adolescentis; BF5, M6) and R (E. faecalis; FF2, PW1) were shown for all isolates fingerprinted from that sample.

Table 1. Details of the different RISA biotypes identified from bacteria isolated from faecal samples of breast- and formula-fed infants

Letters designate distinctive biotypes based on banding patterns obtained from HpaII digestion of amplified 16S-23S rRNA ISRs of faecal isolates selected randomly from Beerens agar plates; superscript numbers represent the number of isolates that displayed each banding profile (maximum of 10 per sample). NC, No colonies were obtained on Beerens plates; NS, no sample collected; -, sample was not processed for cultivation. END, study end.

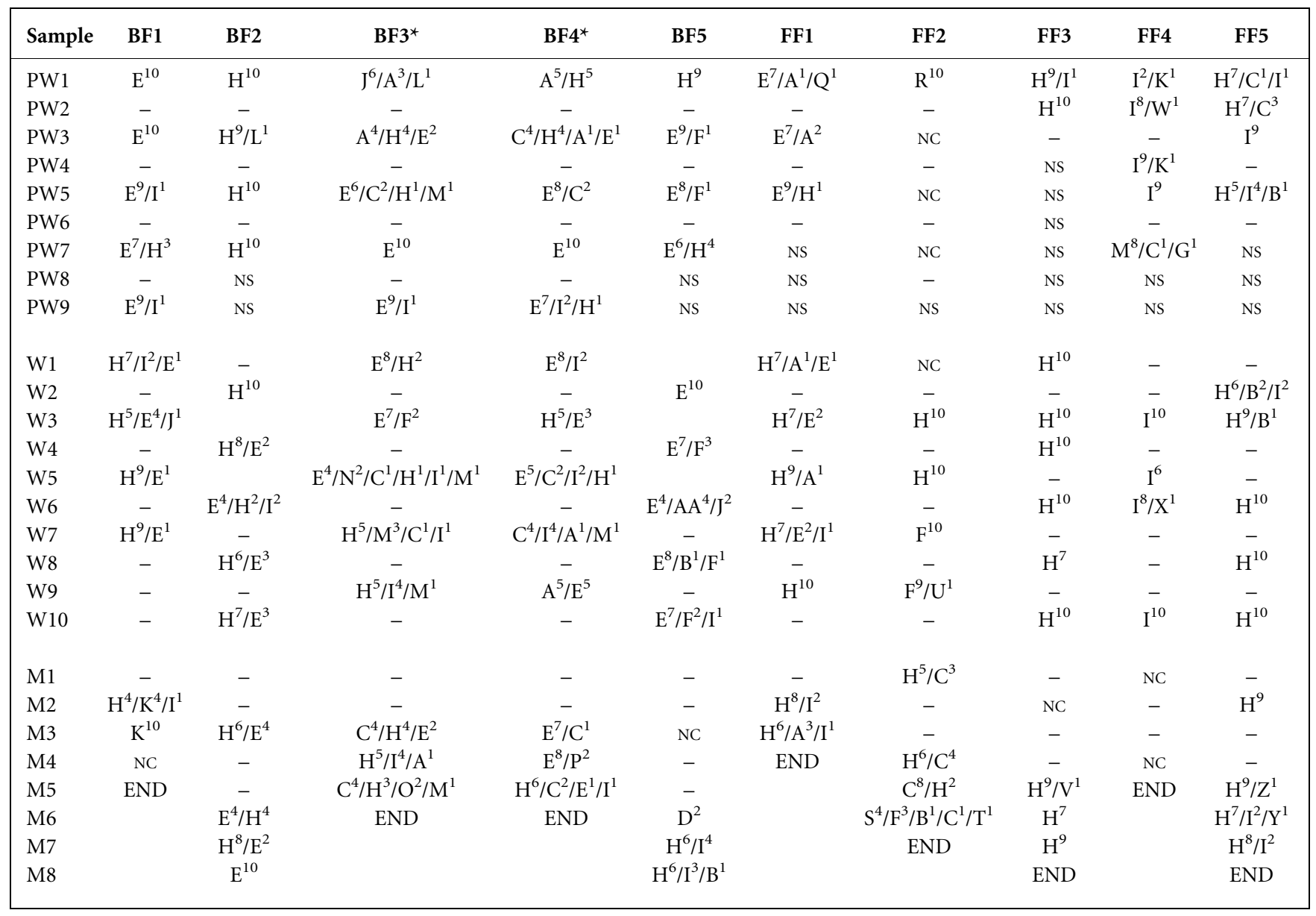

${ }^{\star}$ Identical twins. 
Table 2. Details of bacteria profiled using RISA

\begin{tabular}{|c|c|c|c|c|c|c|c|}
\hline \multirow[t]{2}{*}{ Profile } & \multicolumn{4}{|c|}{ Strain } & \multicolumn{3}{|c|}{ Closest phylogenetic relative } \\
\hline & Name & $\begin{array}{l}\text { Source } \\
\text { infant }\end{array}$ & $\begin{array}{l}\text { 16S rRNA gene } \\
\text { sequence }(\mathrm{nt})\end{array}$ & $\begin{array}{c}\text { GenBank } \\
\text { accession no. }\end{array}$ & Name (GenBank accession no.) & $\begin{array}{c}\text { Sequence similarity (\%) (no. } \\
\text { of nt compared, mismatched } \\
\text { nt, unmatched nt })^{\star}\end{array}$ & Origin $\dagger$ \\
\hline A & LCR1 & BF3 & 1462 & HQ259718 & B. dentium ATCC $27534^{\mathrm{T}}(\mathrm{D} 86183)$ & $99.5(1463,6,1)$ & Dental caries \\
\hline B & LCR2 & BF5 & 1461 & HQ259729 & B. longum subsp. infantis ATCC $15697^{\mathrm{T}}$ (D86184) & $99.9(1461,1,0)$ & Intestine of infant \\
\hline $\mathrm{C}$ & LCR3 & FF2 & 1419 & HQ259738 & B. pseudocatenulatum JCM $1200^{\mathrm{T}}$ (D86187) & $100(1419,0,0)$ & Infant faeces \\
\hline $\mathrm{D}$ & LCR4 & BF5 & 1460 & HQ259739 & B. adolescentis YIT $4011^{\mathrm{T}}$ (AB437354) & $98.3(1463,20,3)$ & Intestine of adult \\
\hline $\mathrm{E}$ & LCR5 & BF1 & 1393 & HQ259740 & B. breve ATCC $15700^{\mathrm{T}}(\mathrm{AB} 006658)$ & $99.9(1393,1,0)$ & Intestine of infant \\
\hline $\mathrm{F}$ & LCR6 & BF5 & 1460 & HQ259741 & B. longum subsp. longum ATCC $15707^{\mathrm{T}}$ (M58739) & $99.8(1447,2,1)$ & Intestine of adult \\
\hline G & LCR7 & FF4 & 1459 & HQ259742 & B. bifidum KCTC $3202^{\mathrm{T}}$ (U25951) & $100(1459,0,0)$ & Stool of breast-fed infant \\
\hline $\mathrm{H}$ & LCR8 & $\mathrm{BF} 2$ & 1362 & HQ259743 & B. longum subsp. infantis ATCC $15697^{\mathrm{T}}$ (D86184) & $99.8(1362,6,0)$ & Intestine of infant \\
\hline I & LCR9 & FF4 & 1469 & HQ259744 & B. bifidum KCTC $3202^{\mathrm{T}}$ (U25951) & $100(1469,0,0)$ & Stool of breast-fed infant \\
\hline J & LCR10 & BF5 & 1406 & HQ259719 & B. breve ATCC $15700^{\mathrm{T}}(\mathrm{AB} 006658)$ & $99.9(1406,1,0)$ & Intestine of infant \\
\hline K & LCR11 & $\mathrm{BF} 1$ & 1451 & HQ259720 & B. bifidum KCTC $3202^{\mathrm{T}}$ (U25951) & $100(1451,0,0)$ & Stool of breast-fed infant \\
\hline $\mathrm{L}$ & LCR12 & BF2 & 1481 & HQ259721 & Staphylococcus pasteuri ATCC $51129^{\mathrm{T}}$ (AB009944) & $99.5(1475,8,0)$ & Human vomit \\
\hline M & LCR13 & $\mathrm{BF} 3$ & 1498 & HQ259722 & Lactobacillus casei subsp. casei ATCC $393^{\mathrm{T}}$ (D16551) & $99.1(1499,11,2)$ & Cheese \\
\hline $\mathrm{N}$ & LCR14 & $\mathrm{BF} 3$ & 1484 & HQ259723 & S. pasteuri ATCC $51129^{\mathrm{T}}$ (AB009944) & $99.8(1484,4,2)$ & Human vomit \\
\hline $\mathrm{O}$ & LCR15 & BF3 & 1479 & HQ259724 & Carnobacterium divergens DSM $20623^{\mathrm{T}}$ (M58816) & $99.9(1472,1,1)$ & Vacuum-packed minced beef \\
\hline $\mathrm{P}$ & LCR16 & BF4 & 1489 & HQ259725 & $\begin{array}{l}\text { Lactobacillus delbrueckii subsp. delbrueckii DSM } \\
20074^{\mathrm{T}} \text { (M58814) }\end{array}$ & $99.6(1480,6,0)$ & Sour grain mash \\
\hline Q & LCR17 & FF1 & 1486 & HQ259726 & E. faecalis JCM $5803^{\mathrm{T}}(\mathrm{Y} 18293)$ & $100(1448,0,0)$ & Faeces \\
\hline $\mathrm{R}$ & LCR18 & FF2 & 1379 & HQ259727 & E. faecalis JCM $5803^{\mathrm{T}}(\mathrm{Y} 18293)$ & $100(1379,0,0)$ & Faeces \\
\hline$S$ & LCR19 & FF2 & 1445 & HQ259728 & Dorea longicatena $111-35^{\mathrm{T}}(\mathrm{AJ} 132842)$ & $99.7(1347,2,3)$ & Human faeces \\
\hline $\mathrm{T}$ & LCR20 & FF2 & 1433 & HQ259730 & Collinsella aerofaciens ATCC $25986^{\mathrm{T}}$ (AB011816) & $98.4(1433,18,5)$ & Human faeces \\
\hline $\mathrm{U}$ & LCR21 & FF2 & 1498 & HQ259731 & $\begin{array}{l}\text { Lactobacillus plantarum subsp. plantarum JCM } 1149^{\mathrm{T}} \\
\text { (D79210) }\end{array}$ & $99.9(1498,3,0)$ & Pickled cabbage \\
\hline $\mathrm{V}$ & LCR22 & FF3 & 1466 & HQ259732 & B. animalis subsp. lactis DSM $10140^{\mathrm{T}}$ (AB050136) & $100(1464,0,0)$ & Rat faeces \\
\hline $\mathrm{W}$ & LCR23 & FF4 & 1443 & HQ259733 & Clostridium baratii ATCC $27638^{\mathrm{T}}$ (X68174) & $99.9(1443,2,0)$ & Canine renal epithelioma \\
\hline $\mathrm{X}$ & LCR24 & FF4 & 1335 & HQ259735 & Clostridium clostridioforme ATCC $25537^{\mathrm{T}}$ (M59089) & $93.8(1335,79,3)$ & Calf rumen \\
\hline Y & LCR25 & FF5 & 1476 & HQ259734 & Clostridium innocuum ATCC $14501^{\mathrm{T}}$ (M23732) & $98.6(1476,19,0)$ & Appendicular abscess \\
\hline $\mathrm{Z}$ & LCR26 & FF5 & 1471 & HQ259736 & B. animalis subsp. lactis DSM $10140^{\mathrm{T}}$ (AB050136) & $100(1471,0,0)$ & Rat faeces \\
\hline AA & LCR27 & BF5 & 1444 & HQ259737 & B. longum subsp. longum ATCC $15707^{\mathrm{T}}$ (M58739) & $99.9(1444,2,1)$ & Intestine of adult \\
\hline
\end{tabular}

*Initial strain identification was based upon WU-BLAST2 searches (http://www.ebi.ac.uk/Tools/blast2/nucleotide.html) followed by comparison with an in-house database of type-strain sequences. Strains were aligned with sequences of type strains of species with validly published names and sequence similarity was calculated based upon comparisons of sequences of the same length. $\dagger$ Information retrieved from www.dsmz.de. 
Six of the seven biotypes seen in BF4 were also observed for BF3 (twin). However, a further five biotypes were found from BF4 (two of which were harboured by BF5). Of the biotypes isolated from multiple infants, only profile $\mathrm{J}(B$. breve) was isolated from one feeding group and not the other.

In general, RISA and Bifidobacterium-specific DGGE results were consistent, even though the two methods employed here target different regions of the rRNA genes. For example, a large number of bands was seen in the Bifidobacterium-specific DGGE profiles for BF3, and 11 distinct biotypes were identified by RISA. RISA is based on typing of bacteria isolated on a so-called selective medium and not all isolates we obtained were bifidobacteria (1045 of 1089 isolates were from the genus Bifidobacterium), although the predominant biotype isolated from each sample was generally bifidobacteria. This is consistent with the findings of Turroni et al. (2009a), who identified that only 704 of the 900 isolates they cultured by using a 'selective' Bifidobacterium medium were actually Bifidobacterium. While our findings suggest Beerens agar may be more 'selective' than mupirocin-based selective medium, Turroni et al. (2009a) examined mucosal and faecal samples from different age groups (infants, adolescents and adults). Unlike Turroni and colleagues, very few $B$. adolescentis and $B$. animalis subsp. lactis biotypes were cultured in this study, and a much greater proportion of isolates from the genus Bifidobacterium were $B$. breve [27.8\%, compared with $2 \%$ of infant Bifidobacterium isolates observed by Turroni et al. (2009a), although $>90 \%$ of these were from breast-fed infants].

RISA profile $\mathrm{E}$ (B. breve) was observed in all breast-fed infants and one formula-fed infant (FF1). The Bifidobacterium-specific DGGE profiles were consistent with this finding, in that the same infants displayed a band consistent with that of $B$. breve in their Bifidobacteriumspecific DGGE profiles. However, RISA contained the $B$. breve-like profile in $\mathrm{W}$ phase samples of $\mathrm{FF} 1$, yet no $B$. breve-like band was seen in the corresponding Bifidobacterium-specific DGGE profile for these samples. This indicated that the use of more than one molecular method is necessary during comparison studies. The most commonly observed Bifidobacterium species in this study were consistent with those seen previously, namely $B$. breve, $B$. bifidum and B. longum subsp. infantis (Mackie et al., 1999; Matsuki et al., 1999; Fanaro et al., 2003; Sakata et al., 2005; Mitsou et al., 2008; Klaassens et al., 2009). However, B. breve did not appear to be a common member of the formula-fed infants' faecal Bifidobacterium population; this was evident in DGGE profiles of only two of the seven formula-fed infants in this study (and the predominant biotype isolated during PW phase for FF1; isolation work was not performed for FF7).

The current data demonstrated that, in general, the Bifidobacterium component (relative abundance and diversity) of the infant faecal microbiota has been under- represented in recent metagenomic studies investigating the human microbiome (Palmer et al., 2007; Koenig et al., 2010; Vaishampayan et al., 2010). Indeed, Koenig and colleagues' comparison of compositional and functional data from a 2.5-year case study of the faecal microbiome of a breast-fed infant highlighted inconsistencies between the 16S rRNA gene data and those generated from shotgun sequencing with subsequent taxonomic assignment of genes, suggesting '16S rRNA gene primer bias' was the most likely explanation for the discrepancy (Koenig et al., 2010). This could also explain the relative lack of abundance of bifidobacteria (and/or Actinobacteria) in metagenomic studies of the infant gut microbiota, such as was seen during the longitudinal study carried out by Palmer et al. (2007). However, the data from our study (herein and in Roger \& McCartney, 2010) generally corroborate the global observations from the longitudinal infant studies performed by Palmer et al. (2007) and Koenig et al. (2010), i.e. the infant gut microbiota develops in a step-wise fashion with increased diversity over time and that life events (including diet) affect the bacterial succession of the infant microbiome (with initial weaning being a transitional period). In addition, longitudinal investigation of the infant Bifidobacterium population concurs with previous microbiomic studies in relation to both inter-individual variations and distinction between the microbiota of breast- and formula-fed infants.

Interestingly, taxonomic assignment of the whole gene sets from shotgun libraries of human faecal microbiome analyses of 13 Japanese individuals (including four unweaned infants and two weaned children) demonstrated a Bifidobacterium component for all but one microbiome (that of a 7-month-old unweaned infant; F1 U) (Kurokawa et al., 2007). Although the overall proportion of proteincoding genes assigned taxonomic characterization was low (generally $<50 \%$ ), the relative proportions of Bifidobacterium across the age groups was consistent with the consensus view of the human Bifidobacterium population associated with life stage, with Bifidobacterium being the main constituent of the unweaned infants' microbiome, but a less predominant constituent of the microbiome of older infants and adults. Kurokawa et al. (2007) also mapped their shotgun reads to the available Bifidobacterium genome sequences in an effort to examine the Bifidobacterium component of the human gut microbiome. Ten of the reads from F1-U (total reads=82 523) mapped to Bifidobacterium genome sequences. However, over $50 \%$ of the reads from two of the other unweaned infants mapped with Bifidobacterium genome sequences, with $>90 \%$ of these mapped to a single Bifidobacterium species: $B$. breve or $B$. longum (which correlates with Bifidobacterium species identification of two of the three common biotypes from breast-fed infants in the present study). Fewer reads from weaned infants' microbiomes mapped to Bifidobacterium genome sequences (1-3\%) and comprised multiple Bifidobacterium species. Vaishampayan et al. (2010) employed microbiomic analysis of the 
Bifidobacterium population similar to that described by Turroni et al. (2009b) and found a single Bifidobacterium phylotype (most closely related to an oral Bifidobacterium strain and B. adolescentis) from the infant faecal sample at 11 months of age (no Bifidobacterium sequences were obtained from the sample taken 1 month after delivery). Overall, the Bifidobacterium data from the current study are in general agreement with those from these two metagenomic studies, demonstrating that the Bifidobacterium populations in the infant gut microbiota are relatively simple and these increase in diversity following the introduction of solid food into the diet.

In summary, Bifidobacterium-specific DGGE and RISA of isolates both show that breast-fed infants generally harboured a greater number of bands/biotypes compared with formula-fed infants during the first 18 months of life. Taken together with the quantitative data presented by Roger \& McCartney (2010), the data from this longitudinal study demonstrate that breast-fed infants harbour both higher levels/a predominance of Bifidobacterium and a more diverse Bifidobacterium population than formula-fed infants. Moreover, the diversity of the Bifidobacterium population appears to increase with the start of weaning, particularly in the breast-fed group. Inter-individual differences were evident in the Bifidobacterium population of infants, although common culturable Bifidobacterium biotypes were observed, and the greatest similarity was seen in the Bifidobacterium populations of the twins. This is the first longitudinal study, to our knowledge, that monitors the Bifidobacterium populations of infants and the impact of weaning on this bacterial group in an effort to determine the transition from the infant Bifidobacterium population to the adult Bifidobacterium population. Weaning correlated with increased diversity, yet the predominant Bifidobacterium species remained those more commonly associated with the infant microbiota than that of adults. As such, further work is necessary to fully elucidate the bacterial succession and development of the adult Bifidobacterium community.

\section{ACKNOWLEDGEMENTS}

We would like to acknowledge the financial support of Wyeth Pharmaceuticals, Philadelphia, USA (Martin Kullen, PhD).

\section{REFERENCES}

Beerens, H. (1991). Detection of bifidobacteria by using propionic acid as a selective agent. Appl Environ Microbiol 57, 2418-2419.

Dai, D. \& Walker, W. A. (1999). Protective nutrients and bacterial colonization in the immature human gut. Adv Pediatr 46, 353-382.

Dinoto, A., Marques, T. M., Sakamoto, K., Fukiya, S., Watanabe, J., Ito, S. \& Yokota, A. (2006). Population dynamics of Bifidobacterium species in human feces during raffinose administration monitored by fluorescence in situ hybridization-flow cytometry. Appl Environ Microbiol 72, 7739-7747.
Fanaro, S., Chierici, R., Guerrini, P. \& Vigi, V. (2003). Intestinal microflora in early infancy: composition and development. Acta Paediatr Suppl 91, 48-55.

Harmsen, H. J. M., Elfferich, P., Schut, F. \& Welling, G. W. (1999). A $16 \mathrm{~S}$ rRNA-targeted probe for detection of lactobacilli and enterococci in faecal samples by fluorescent in situ hybridization. Microb Ecol Health Dis 11, 3-12.

Harmsen, H. J. M., Wildeboer-Veloo, A. C., Grijpstra, J., Knol, J., Degener, J. E. \& Welling, G. W. (2000a). Development of $16 \mathrm{~S}$ rRNAbased probes for the Coriobacterium group and the Atopobium cluster and their application for enumeration of Coriobacteriaceae in human feces from volunteers of different age groups. Appl Environ Microbiol 66, 4523-4527.

Harmsen, H. J. M., Wildeboer-Veloo, A. C., Raangs, G. C., Wagendorp, A. A., Klijn, N., Bindels, J. G. \& Welling, G. W. (2000b). Analysis of intestinal flora development in breast-fed and formula-fed infants by using molecular identification and detection methods. J Pediatr Gastroenterol Nutr 30, 61-67.

Hopkins, M. J., Macfarlane, G. T., Furrie, E., Fite, A. \& Macfarlane, S. (2005). Characterisation of intestinal bacteria in infant stools using real-time PCR and Northern hybridisation analyses. FEMS Microbiol Ecol 54, 77-85.

Hoppu, U., Kalliomaki, M., Laiho, K. \& Isolauri, E. (2001). Breast milk - immunomodulatory signals against allergic diseases. Allergy 56 (Suppl. 67), 23-26.

Howie, P. W. (2002). Protective effect of breastfeeding against infection in the first and second six months of life. Adv Exp Med Biol 503, 141-147.

Isolauri, E., Arvola, T., Sutas, Y., Moilanen, E. \& Salminen, S. (2000). Probiotics in the management of atopic eczema. Clin Exp Allergy 30, 1604-1610.

Jiang, T., Mustapha, A. \& Savaiano, D. A. (1996). Improvement of lactose digestion in humans by ingestion of unfermented milk containing Bifidobacterium longum. J Dairy Sci 79, 750-757.

Kabadjova, P., Dousset, X., Le Cam, V. \& Prevost, H. (2002). Differentiation of closely related Carnobacterium food isolates based on 16S-23S ribosomal DNA intergenic spacer region polymorphism. Appl Environ Microbiol 68, 5358-5366.

Kimura, K., McCartney, A. L., McConnell, M. A. \& Tannock, G. W. (1997). Analysis of fecal populations of bifidobacteria and lactobacilli and investigation of the immunological responses of their human hosts to the predominant strains. Appl Environ Microbiol 63, 33943398.

Kirjavainen, P. V., Arvola, T., Salminen, S. J. \& Isolauri, E. (2002). Aberrant composition of gut microbiota of allergic infants: a target of bifidobacterial therapy at weaning? Gut 51, 51-55.

Klaassens, E. S., Boesten, R. J., Haarman, M., Knol, J., Schuren, F. H., Vaughan, E. E. \& de Vos, W. M. (2009). Mixed-species genomic microarray analysis of fecal samples reveals differential transcriptional responses of bifidobacteria in breast- and formula-fed infants. Appl Environ Microbiol 75, 2668-2676.

Klijn, A., Mercenier, A. \& Arigoni, F. (2005). Lessons from the genomes of bifidobacteria. FEMS Microbiol Rev 29, 491-509.

Koenig, J. E., Spor, A., Scalfone, N., Fricker, A. D., Stombaugh, J., Knight, R., Angenent, L. T. \& Ley, R. E. (2010). Succession of microbial consortia in the developing infant gut microbiome. Proc Natl Acad Sci U S A, doi: 10.1073/pnas.1000081107.

Kurokawa, K., Itoh, T., Kuwahara, T., Oshima, K., Toh, H., Toyoda, A., Takami, H., Morita, H., Sharma, V. K. \& other authors (2007). Comparative metagenomics revealed commonly enriched gene sets in human gut microbiome. DNA Res 14, 169-181. 
Leblond-Bourget, N., Philippe, H., Mangin, I. \& Decaris, B. (1996). $16 \mathrm{~S}$ rRNA and $16 \mathrm{~S}$ to $23 \mathrm{~S}$ internal transcribed spacer sequence analyses reveal inter- and intraspecific Bifidobacterium phylogeny. Int J Syst Bacteriol 46, 102-111.

Liepke, C., Adermann, K., Raida, M., Magert, H. J., Forssmann, W. G. \& Zucht, H. D. (2002). Human milk provides peptides highly stimulating the growth of bifidobacteria. Eur J Biochem 269, 712-718.

López-Alarcón, M., Villalpando, S. \& Fajardo, A. (1997). Breastfeeding lowers the frequency and duration of acute respiratory infection and diarrhea in infants under six months of age. J Nutr 127, 436-443.

Mackie, R. I., Sghir, A. \& Gaskins, H. R. (1999). Developmental microbial ecology of the neonatal gastrointestinal tract. Am J Clin Nutr 69, 1035S-1045S.

Matsuki, T., Watanabe, K., Tanaka, R., Fukuda, M. \& Oyaizu, H. (1999). Distribution of bifidobacterial species in human intestinal microflora examined with 16S rRNA-gene-targeted species-specific primers. Appl Environ Microbiol 65, 4506-4512.

Matsuki, T., Watanabe, K., Fujimoto, J., Kado, Y., Takada, T., Matsumoto, K. \& Tanaka, R. (2004). Quantitative PCR with $16 \mathrm{~S}$ rRNA-gene-targeted species-specific primers for analysis of human intestinal bifidobacteria. Appl Environ Microbiol 70, 167-173.

McCartney, A. L., Wenzhi, W. \& Tannock, G. W. (1996). Molecular analysis of the composition of the bifidobacterial and lactobacillus microflora of humans. Appl Environ Microbiol 62, 4608-4613.

McClellan, D. L., Griffen, A. L. \& Leys, E. J. (1996). Age and prevalence of Porphyromonas gingivalis in children. J Clin Microbiol 34, 20172019.

Mitsou, E. K., Kirtzalidou, E., Oikonomou, I., Liosis, G. \& Kyriacou, A. (2008). Fecal microflora of Greek healthy neonates. Anaerobe 14, 94101.

Mitsuoka, T. (1996). Intestinal flora and human health. Asia Pac J Clin Nutr 5, 2-9.

Noda, H., Akasaka, N. \& Ohsugi, M. (1994). Biotin production by bifidobacteria. J Nutr Sci Vitaminol (Tokyo) 40, 181-188.

Palmer, C., Bik, E. M., Digiulio, D. B., Relman, D. A. \& Brown, P. O. (2007). Development of the human infant intestinal microbiota. PLoS Biol 5, e177.

Roger, L. C. \& McCartney, A. L. (2010). Longitudinal investigation of the faecal microbiota of healthy full-term infants using fluorescence in situ hybridization and denaturing gradient gel electrophoresis. Microbiology 156, 3317-3328.

Saavedra, J. M., Bauman, N. A., Oung, I., Perman, J. A. \& Yolken, R. H. (1994). Feeding of Bifidobacterium bifidum and Streptococcus thermophilus to infants in hospital for prevention of diarrhoea and shedding of rotavirus. Lancet 344, 1046-1049.
Sakata, S., Tonooka, T., Ishizeki, S., Takada, M., Sakamoto, M., Fukuyama, M. \& Benno, Y. (2005). Culture-independent analysis of fecal microbiota in infants, with special reference to Bifidobacterium species. FEMS Microbiol Lett 243, 417-423.

Salonen, A., Nikkilä, J., Jalank-Tuovinen, J., Immonen, O., RajilicStojanovic, M., Kekkonen, R. A., Palva, A. \& de Vos, W. M. (2010). Comparative analysis of fecal DNA extraction methods with phylogenetic microarray: effective recovery of bacterial and archaeal DNA using mechanical cell lysis. J Microbiol Methods 81, 127-134.

Satokari, R. M., Vaughan, E. E., Akkermans, A. D., Saarela, M. \& de Vos, W. M. (2001). Bifidobacterial diversity in human feces detected by genus-specific PCR and denaturing gradient gel electrophoresis. Appl Environ Microbiol 67, 504-513.

Turroni, F., Foroni, E., Pizzetti, P., Giubellini, V., Ribbera, A., Merusi, P., Cagnasso, P., Bizzarri, B., de'Angelis, G. L. \& other authors (2009a). Exploring the diversity of the bifidobacterial population in the human intestinal tract. Appl Environ Microbiol 75, 1534-1545.

Turroni, F., Marchesi, J. R., Foroni, E., Gueimonde, M., Shanahan, F., Magolles, A., van Sinderen, D. \& Ventura, M. (2009b). Microbiomic analysis of the bifidobacterial population in the human distal gut. ISME J 3, 745-751.

Vaishampayan, P. A., Kuehl, J. V., Froula, J. L., Morgan, J. L., Ochman, H. \& Francino, M. P. (2010). Comparative metagenomics and population dynamics of the gut microbiota in mother and infant. Genome Biol Evol 2, 53-66.

van Verseveld, H. W. \& Röling, W. F. M. (2004). Cluster analysis and statistical comparison of molecular community profile data. In Molecular Microbial Ecology Manual, 2nd edn, vol. 2, pp. 1373-1396. Edited by G. A. Kowalchuk, F. J. de Bruijn, I. M. Head, A. D. L. Akkermans \& J. D. van Elsas. The Netherlands: Kluwer Academic Publishers.

Ventura, M., Elli, M., Reniero, R. \& Zink, R. (2001). Molecular microbial analysis of Bifidobacterium isolates from different environments by the species-specific amplified ribosomal DNA restriction analysis (ARDRA). FEMS Microbiol Ecol 36, 113-121.

Ventura, M., Canchaya, C., Zhang, Z., Bernini, V., Fitzgerald, G. F. \& van Sinderen, D. (2006). How high G + C Gram-positive bacteria and in particular bifidobacteria cope with heat stress: protein players and regulators. FEMS Microbiol Rev 30, 734-759.

Wilson, A. C., Forsyth, J. S., Greene, S. A., Irvine, L., Hau, C. \& Howie, P. W. (1998). Relation of infant diet to childhood health: seven year follow up of cohort of children in Dundee infant feeding study. BMJ 316, 21-25.

Wold, A. E. \& Adlerberth, I. (2000). Breast feeding and the intestinal microflora of the infant - implications for protection against infectious diseases. Adv Exp Med Biol 478, 77-93.

Edited by: P. W. O'Toole 